\title{
8 Der Ort der Grenze: Verortung und Entortung von Kontroll- und Überwachungspraktiken
}

Entgegen der kartographisch vielfach produzierten und reproduzierten linearen Gestalt von Grenzen, die eine konkrete Lokalisierung visualisiert, betont die wissenschaftliche Literatur den zunehmend entorteten bzw. vielfach verorteten Charakter von Grenze. ${ }^{1}$ Das hängt weniger damit zusammen, dass Grenzverläufe selbst zwischen territorial vermeintlich klar definierten Staaten uneindeutig und umstritten sein können, ${ }^{2}$ sondern vielmehr mit der Aufspaltung von Kontroll- und Überwachungsmaßnahmen in eine Vielzahl einzelner Prozeduren und Routinen, die dem eigentlichen Akt der Grenzüberquerung sowohl vor- wie auch nachgelagert sein können. Prozeduren im Vorfeld umfassen u. a. Visabeantragungen, persönliche Interviews in Konsulaten, Datenbankabfragen, Vermögensnachweise, Prozeduren im Hinterland wie z. B. verdachtsunabhängige Kontrollen. All das kann zunächst durchaus als Zeichen der Enträumlichung von Grenzen gelesen werden. Diese Enträumlichung geht jedoch mit neuen Verortungen von Kontrollund Überwachungspraktiken einher, die jetzt allerdings räumlich fragmentiert

1 Laube, Wohin mit der Grenze?; W. Walters, „Border/Control“, European Journal of Social Theory 9 (2006) 2, S. 187-203.

2 Auch zwischen Mitgliedsstaaten der Europäischen Union gibt es strittige Grenzfragen, beispielsweise zwischen den Niederlanden und Deutschland im Gebiet des Emsästuars. Im Alltag hat sich in diesem Fall - u. a. beim Bau des Rijksweg 7 inkl. der Raststätte Bunderneuland - ein pragmatischer Umgang durchgesetzt. Potenziell konfliktträchtig sind ungeklärte Grenzverläufe v. a. dann, wenn in dem strittigen Gebiet Rohstoffe vermutet werden oder bereits nachgewiesen wurden, wie z. B. im Gebiet der ungeklärten Seegrenze zwischen Bulgarien und Rumänien im Schwarzen Meer. Auch die 1892 im Ligurischen Meer zwischen Sardinien und Korsika festgelegte und vor wenigen Jahren neu verhandelte Seegrenze zwischen Italien und Frankreich ist aktuell (2018) wieder umstritten, weil dort ertragreiche Fanggebiete für die Roten Riesengarnelen liegen (https://www.esteri.it/mae/en/sala_stampa/archivionotizie/comunicati/nota-della-farnesina-sul la-delimitazione.html [abgerufen am 3. April 2018]). Lange Zeit umstritten war auch die Schlangeninsel im Schwarzen Meer und die damit verbundene Aufteilung des schwarzmeerischen Kontinentalsockels zwischen Rumänien und der Ukraine; dieser Fall wurde 2009 durch einen Schiedsspruch des Internationalen Gerichtshofs beigelegt. Einen ähnlichen Fall gibt es im Nordatlantik: Der mit einem Durchmesser von 30 Metern eher kleine, sturmumtoste Felsen Rockall ist für sich genommen nicht sonderlich attraktiv, wohl aber die Fischereirechte sowie die Rechte zur Ausbeutung der Erdölvorkommen innerhalb der 200-Seemeilen-Zone um die Insel, weshalb sich Island, Irland, Großbritannien sowie - stellvertretend für die Färöer-Inseln - Dänemark seit Jahren um die einsam aufragende Felsnadel am Rande des europäischen Kontinentalsockels streiten. (Da Großbritannien mittlerweile allerdings anerkannt hat, dass Rockall nicht bewohnbar ist, spielt die Frage der Fischereirechte aktuell keine Rolle mehr.). 
sind. Diese Verschiebungen und Neuverräumlichungen sind sowohl Folge wie auch Mittel des Einfügens grenzsichernder Praktiken in umfassende und oftmals ihrer konkreten Zielsetzung enthobenen Prozesse der Versicherheitlichung. ${ }^{3}$

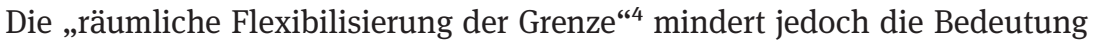
der Grenzlinie keineswegs, vielmehr bleibt diese in zweifacher Hinsicht zentral. Eine Dezentralisierung von Kontroll- und Überwachungstechniken, ein räumliches Aufspannen der Grenzfunktionen bis hin zu ihrer wahrgenommenen Ubiquität, und auch ein Überlagern von Souveränitäten bedeuten nicht, dass die Linearität der Grenze bedeutungslos wird.

Zum einen ändert ihr Überqueren fundamental den Status desjenigen, der sie überschritten (oder überwunden) hat: Von der Staatsbürger^in zur Besucher^in, zum/zur Fremden, zum/zur Migrant*in, zur Saisonarbeiter^in, zum/zur Geflüchteten, zum/zur Illegalen, zu einer anderen Position, die wesentlich vom geopolitischen Verhältnis zwischen Herkunfts- und Zielstaat abhängt. Die territoriale Logik des Raumformats Nationalstaat, zu dem der Körper ins Verhältnis gesetzt wird, sieht zunächst einmal keine andere Möglichkeit als die des Innen/ Außen - dieser oder eben jener Staat - vor. Praktisch mag das aber durchaus anders aussehen; da gibt Niemandsländer, neutrale Zonen, Zonen unklarer und ungeklärter Grenzverläufe oder auch Orte, deren Kontrolle aufwändig oder technisch schwierig ist, so dass zunächst die Anwesenheit von Menschen nicht festgestellt wird (z.B. auf See, in Wüstengebieten). Die Bewegung von Körpern im Verhältnis zum (definierten, gesicherten, eindeutig zugeordneten) Territorium ist keineswegs trivial. Dies schlägt sich nicht zuletzt in permanenten Bemühungen nieder, die technischen Möglichkeiten des Entdeckens der Körper illegalisierter Menschen auszudehnen, sowie in der Bereitschaft, in Sicherheitstechnologien zu investieren ${ }^{5}$. Rechtsbegriffe wie die „Fiktion der Nichteinreise“6 - ein in Asylverfahren an Flughäfen regelmäßig verwendeter Begriff - spiegeln dieses Spannungsverhältnis wider: Auch wenn der Körper die Grenze zum Territorium bereits überquert hat, gilt er als außerhalb befindlich solange die zuständigen Grenzschutz- und Zollkontrollen nicht passiert wurden. Diese Aufspaltung ermöglicht dann wiederum neue räumliche Konstruktionen, wie die der Transitzonen, die

3 Belina, Miggelbrink, „Am Ostrand des „wettbewerbsfähigsten Wirtschaftsraums der Welt“.

4 Laube, Wohin mit der Grenze?.

5 B. C. Newell, R. Gomez und V. Guajardo, „Sensors, Cameras, and the New 'Normal' in Clandestine Migration. How Undocumented Migrants Experience Surveillance at the US-Mexico Border“, Surveillance \& Society 15 (1), 2017, S. S. 21- 41.

6 B. Nieswand, „Die Transitzone und die Fiktion der Nichteinreise. Das Flughafenasylverfahren im Zwielicht von Normalität und Ausnahme“, in: J. Oltmer (Hg.), Migrationsregime vor Ort und lokales Aushandeln von Migration, Wiesbaden: Springer-Fachmedien, 2018, S. 345-376. 
gewissermaßen zwischen einen migrierenden Menschen und den Staat geschoben werden.

Zum anderen macht die Markierung der Grenze die Macht des Staates sichtbar und symbolisiert sie. Das geschieht mancherorts durch simple Grenzpfosten und Warnschilder, an anderen Orten dagegen durch massive, in Stahl, Beton und Stacheldraht gefasste Barrieren. In beiden Fällen markiert das grenzbezogene Zeichen das Ende der legalen Bewegung und zugleich die Macht des Staates, der diese Bewegung kontrolliert und überwacht. Die Grenzlinie ist der Ort, an dem das Grenzregime materiell und symbolisch kulminiert und daher der Ort, an dem der Staat seine Fähigkeit zur Kontrolle der Bewegung demonstriert; sie ist der Ort, an dem der Staat seine Souveränität inszeniert. ${ }^{7}$

Im Kontext der Migrations- und Flüchtlingspolitik der EU versteht De Genova ${ }^{8}$ die US-amerikanisch-mexikanische Grenze als Bühne, auf der die Inszenierung des „illegalen Fremden“ aufgeführt werde und spricht vom border spectacle. Die relative Unsichtbarkeit des Gesetzes bedürfe Strategien des Sichtbarmachens seiner Vollstreckung; diese Strategien manifestierten sich in Mauern und Zäunen, die nicht nur physische Barrieren darstellen, die kaum zu überwinden seien, sondern stellten zugleich Symbole für die Fähigkeit und den Willen des Staates dar, sein souveränes Recht zur Kontrolle seiner territorialen Grenze durchzusetzen. Anders ausgedrückt: Die Staatsgrenze als lineares Gebilde wird durch Prozesse, Organisationen und Infrastrukturen stabilisiert, von denen viele „überall“ lokalisiert sein können. Weder Server noch Konsulate müssen „an der Grenze“ lokalisiert sein; sog. Hinterlandkontrollen sind in vielen europäischen Ländern auf dem gesamten Territorium möglich und damit keineswegs an die Grenze gebunden. Der räumlichen Dispersion der Ausübung von Kontrolle und Überwachung muss dann aber auf der symbolischen Ebene die Inszenierung von Grenze als konkretem Ort des Vollzugs staatlicher Gewalt entgegenwirken.

Die symbolische Funktion der Grenze als Ort der Inszenierung der Vollstreckung staatlicher Souveränität wird auf ganz unterschiedliche Weise genutzt: Sie zeigt sich beispielsweise in den technik-dominierten, infografischen Spielzeug-

7 Im Streit um die europäische Flüchtlingspolitik wurde die Demonstration der Möglichkeit territorialer Abschottung ein in symbolischer Hinsicht wichtiger Akt der Wahrung und/oder Rückgewinnung vermeintlicher und tatsächlicher Souveränitätsverluste. An der Beurteilung der Fähigkeit, die territorialen Grenzen zu kontrollieren, wird mithin die „Stärke“ eines Staates ebenso wie sein „Versagen“ (bzw. ein Versagen „der Politik“) gemessen.

8 N. S. De Genova, „Migrant ,Illegality“ and Deportability in Everyday Life“, Annual Review of Anthropology 31 (2002), S. 419-447, hier S. 436. 
landschaften der Europäischen Kommission. In deren Broschüren ${ }^{9}$ werden grenzrelevante Infrastrukturen grafisch verortet, wodurch Grenzsicherung als eine vornehmlich entlang einer linearen Infrastruktur aufgeführte Kontroll- und Überwachungspraxis festgeschrieben wird. Sie zeigt sich ferner in Fernsehserien: Den Abstand zwischen der demonstrativen Inszenierung der Durchsetzung eines Grenzregimes und der relativen Ferne des Alltagslebens der meisten Staatsbürger^innen von diesem Geschehen überbrücken seit einigen Jahren nicht mehr nur Berichterstattungen in den Nachrichten und populär-literarische Darstellungen wie beispielsweise Tortilla Curtain von T C Boyle, sondern auch dokumentarisch angelegte Serien (factual television programs), die im Stil einer Telenovela episodenhaft den Alltag an Grenzen erzählen. ${ }^{10}$ Die vermeintlich dokumentarische Perspektive ist, wie Reece Jones ${ }^{11}$ in seiner Analyse der vom National Geographic Channel produzierten Serie Border Wars herausarbeitet, nahezu ausschließlich eine Perspektive der Grenzschützer*innen und fokussiert deren Einsätze anhand möglichst spektakulärer Vorfälle. Die Border Wars fügen sich demnach nahtlos in das Dispositiv der Versicherheitlichung ein, indem sie „Grenze“ als einen endlosen, von Grenzschützerinnen im Dienste der Nation tagtäglich geführten Kampf gegen Bedrohungen inszenieren.

Die symbolische Funktion der materialisierten Grenze wird auch auf kritische Weise in zahlreichen künstlerischen Interventionen genutzt, die sich den Ort der Entfaltung staatlicher Kontroll- und Überwachungspraktiken als den Ort inszenieren, an dem sich dessen Widersprüche, Ausschlüsse und Ungerechtigkeiten herausfordern und anklagen lassen. Beispiele dafür sind die Projekte Borrando la Frontera von Ana Teresa Fernández ${ }^{12}$ und JRs Gigantic Picnic at the US-Mexico

9 European Commission, A Common Information Sharing Environment for the European maritime domain. How data-sharing can increase knowledge, detct illegal activities and save costs. o.0., 2012; https://ec.europa.eu/maritimeaffairs/maritimeday/sites/mare-emd/files/20120521_15_program me_en.pdf, (accessed 24 September 2017).

10 Unter anderem Border Security: Australia's Front Line (Australien, Seven Network, erste Staffel 2004, neue Staffel für 2018 angekündigt), Border Wars (USA, National Geographic Channel, 2010 - 2013), Nothing to Declare UK (Vereinigtes Königreich, 2011), Border Security: Canada's Front Line (Kanada, Force Four Entertainment, National Geographic Channel, 2012). In Alerta Areopuerto inszeniert National Geographic Channel zudem Kontroll- und Überwachungspraxen an Flughäfen u. a. in Kolumbien und Peru.

11 R. Jones, „Border Wars: Narratives and Images of the US-Mexico Border on TV“ in: R. Jones, C. Johnson (Hrsg.), Placing the Border in Everyday Life. Farnham: Ashgate, 2014, S. 185-204.

$12 \mathrm{http}$ //anateresafernandez.com/borrando-la-barda-tijuana-mexico/ (abgerufen am 12. März 2019); Ana Teresa Fernández wird gelegentlich als border artist bezeichnet: https://www.azcen tral.com/story/entertainment/arts/2015/10/07/asu-public-art-mexican-border/73539840/, （abgerufen am 28. Februar 2019). 
Border Fence. ${ }^{13}$ Im europäischen Kontext hat das Paneuropäische Picknick am 19. August 1989 in Sopron, organisiert vom oppositionellen ungarischen Demokratischen Forum und der Paneuropa-Union, einige Aufmerksamkeit erregt und wurde dadurch selbst zu einem performativen Akt der Überwindung von Grenzen. Es ist nicht ganz ohne Ironie, dass am 3. September 2015 im Budapester KeletiBahnhof eine Lok stand, deren Aufschrift an den 25. Jahrestag dieses Frühstücks erinnerte, während gleichzeitig Hunderte geflüchtete und in Budapest gestrandete Menschen in den Bahnhof rannten, um nach Tagen des Wartens auf dem Bahnhofsvorplatz einen Zug zur Ausreise aus Ungarn zu erreichen. Der Gegensatz von medialer Inszenierung von Grenzlinie und Grenzinfrastruktur auf der einen Seite und der Tendenz, Grenzfunktionen immer weiter $\mathrm{zu}$ verstreuen, auszudifferenzieren und an unterschiedlichsten Stellen anzusiedeln auf der anderen, ist also nur ein scheinbarer. Die mediale Inszenierung ist ein zur Entwicklung des Grenzregimes komplementärer Akt, der dessen Fragmentierung auf symbolischer Ebene mindestens partiell wieder zurückzugewinnen versucht.

Ein im Hinblick auf seine sozialweltliche Ordnungsfunktion verallgemeinerter Grenzbegriff wie im vorangehenden Kapitel erläutert, ermöglicht nun einen neuen Blick auf die physische Materialität von Grenzen: Wenn die Bedeutung der physischen Materialität als sozial relevante Form der Durchsetzung und Aufrechterhaltung einer bestimmten Form gesellschaftlicher Ordnung anerkannt wird, kann diese wiederum zum Ausgangspunkt künstlerisch-politischer Interventionen genutzt werden, ${ }^{14}$ die die Kontingenz von Grenzziehungen sichtbar machen. Die wenigen Beispiele zeigen, dass die physische Lokalisierung und Gestalt(ung) der Grenze die durchgesetzte gesellschaftliche Funktion der Grenze garantiert und symbolisiert, weswegen der Grenzraum gleichermaßen der Ort der Manifestation wie auch des (künstlerischen) Infragestellens eines Grenzregimes ist.

13 Fotograf JR (Juste Ridicule) http://www.jr-art.net/ (abgerufen am 28. Februar 2018). Siehe auch die Installation eines riesenhaft vergrößerten Fotos eines Dreamer-Kindes an derselben Grenze: https://www.nytimes.com/2017/09/07/arts/design/jr-artist-mexico-border-wall.html (abgerufen am 28. Februar 2019). Mittlerweile werden Protest Art-Beiträge zur US-amerikanischmexikanischen Grenze aus verschiedenen Webseiten gesammelt, z. B. https://www.voiceofsandi ego.org/topics/arts/the-most-memorable-acts-of-protest-art-at-the-border/ (abgerufen am 28. Februar 2019).

14 Auch die antiAtlas-Projekte, die sich zwischen Wissenschaft, politischer Intervention und Kunst bewegen und sich bewusst klarer Zuordnungen entziehen, können hierzu gerechnet werden; vgl. z. B. https://www.antiatlas-journal.net/anti-atlas/01-border-art-and-the-borders-of-art/ (abgerufen am 24. März 2019). 